\title{
Use of a viewdata system to collect data from a multicentre clinical trial in anaesthesia
}

\author{
H A WALDRON, R F COOKSON
}

\begin{abstract}
The interactive electronic information storage and transmission system PRESTEL was assessed as a method of recording and collecting patient record forms from a multicentre trial in anaesthesia. PRESTEL terminals were provided in anaesthetic centres around Britain and all data handled by this public viewdata service, which connects users by telephone to a central computer. The trial was of a new analgesic supplement, alfentanil, and confirmed more rapid recovery of patients as compared with that after traditional anaesthesia with halothane.

Advantages of the system were manifold and included reducing the need for the trial monitor to visit the trialist, an electronic "mailbox," confidentiality, and the ability immediately to identify violations of study protocol. No participant found the system too difficult to use, though the small keyboard was a source of complaint. Despite the initial cost of the system its utility vastly outweighs traditional methods of data collection.
\end{abstract}

\section{Introduction}

Viewdata is an interactive electronic information storage and transmission system which connects users by telephone to a central computer. British Telecom invented the system and now market PRESTEL, the world's first public viewdata service. Other countries have viewdata systems, although PRESTEL remains by far the largest in terms of breadth and quantity of information and its thousands of customers. In Britain emphasis was placed initially on a nationally available system. By contrast, in Western Germany a more geographically limited service (BILDSCHIRMTEXT) was introduced, emphasising a wide range of services (shopping, banking, etc) of interest to private subscribers. These facilities will gradually be introduced on PRESTEL, although up to now British Telecom have concentrated on the business and professional communities.

In the wake of travel agents and stockbrokers, the medical profession has been identified as a coherent group whose information needs may be amenable to handling by PRESTEL. An early example of this is the joint project between the $B M F$ and Glaxo to publicise job vacancies and the contents page of the journal. PRESTEL compatible computers have become increasingly available in hospitals and the viewdata approach used to provide access to information on drugs and poisons. ${ }^{12}$

In late 1982 Janssen Pharmaceutical Ltd donated a number of PRESTEL terminals to departments of anaesthesia in the United

Janssen Pharmaceutical Ltd, Grove, Wantage, Oxon OX12 0DQ

H A WALDRON, BSC, MSC, research project manager

R F COOKSON, MA, PHD, clinical development manager

Correspondence to: Miss H A Waldron.
Kingdom to facilitate access by anaesthetists to its technical information service. We have investigated the utility of the system "in reverse" to collect data from a multicentre study on the new narcotic analgesic alfentanil.

\section{Multicentre trials}

Registration and marketing of a drug may be achieved after a small number of good quality trials. Nevertheless, the eventual role of a new drug in clinical practice is dependent on, and can therefore only be defined by, its performance characteristics when used routinely by a representative sample of clinicians. Large multicentre trials provide an essential step between controlled studies in centres specialising in clinical research and the routine use of a therapeutic agent after marketing. This step is particularly important in anaesthesia: anaesthetics are not normally given according to a fixed dosage schedule, and usage of drugs may vary widely depending on the anaesthetist's assessment of the patient and on the variable stresses related to the surgical conditions. In order to allow for variations among anaesthetists we organised a multicentre study with as many centres as was practicable, each centre contributing a minimum of 20 patients.

The study was designed to compare speed of recovery after the use of alfentanil and halothane as supplements to methohexitone-nitrous oxide anaesthesia in minor surgical procedures. The volume of data to be collected and the geographical spread of the participating centres made it logical to consider a PRESTEL based system for collecting the patient record forms.

\section{Methods \\ VIEWDATA}

Each participating centre used a PRESTEL terminal comprising a keyboard (Tandata Marketing Ltd) and a red-green-blue monitor (Microvitec) (fig 1). These were connected by addition of a jack socket to the hospital telephone system to allow automatic dialling to the local PRESTEL computer node. To ensure confidentiality each set was registered as a member of a closed user group, and to activate the system once connected to PRESTEL a personal password had to be entered. The nature of the system is such that once a patient record had been entered and dispatched it could be examined only by the trial monitors.

Vismed Ltd, Maidenhead, wrote the software and monitored the record forms as they were received. A menu offered the investigator three choices on access to the reporting system. A summary of the trial protocol (four frames) was available for reference as the first option. The second item allowed transmission of data on individual patients (eight frames per patient). The third choice gave a free format "help" page, which allowed the anaesthetist to type in comments or messages which could then be transmitted instantly to Vismed or the Janssen trial monitor with the opportunity of a response the same day.

The anaesthetist entered data by selecting the appropriate starting point from the menu and then typing details of the patient on a form organised electronically to ensure that sections were completed in a predetermined sequence (fig 2). Different colours were used to separate and identify spaces for data entry and to contrast with headings and instructions. The action of calling the record form automatically recorded the investigator or centre by name and the date and time of data entry. On completion of each form the data were transmitted to the central computer. Incorrectly entered data could be modified easily by the anaesthetist before transmission. The data 


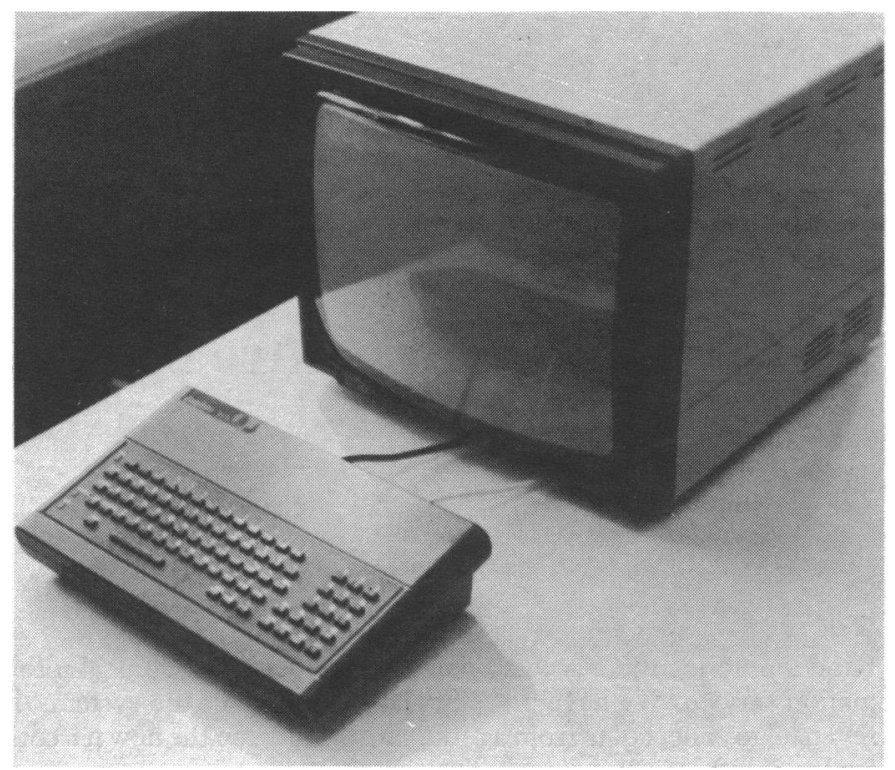

FIG 1-Tandata keyboard and Microvitec monitor.

were stored on the PRESTEL central computer and were available instantly for access by the trial monitor. In this study forms were downloaded on to an Apple IIe computer with an Owl Editel system for sequential checking and validation. Forms were stored as Pascal files on floppy discs. Analysis was performed separately on an IBM System 38.

\section{ANAESTHETIC TECHNIQUE}

Thirty nine anaesthetic centres contributed data to the trial and the protocol was approved by the local ethical committee in each case. The study group comprised 907 patients who underwent minor general, gynaecological, or urinary tract procedures that did not entail cutting the skin. All gave informed consent to the investigation and breathed spontaneously throughout. Within each centre patients were allocated at random to receive either alfentanil or halothane. According to the anaesthetist's preference, patients were either not premedicated or given lorazepam $1 \mathrm{mg}$ by mouth two hours before induction or temazepam $20 \mathrm{mg}$ by mouth one hour before induction.

In the alfentanil group the anaesthetic sequence was intravenous alfentanil $500 \mu$ given slowly, followed immediately by $1 \%$ methohexitone until the eyelash reflex was obtunded. Patients then breathed $66 \%$ nitrous oxide ir oxygen. The anaesthetist gave methohexitone in increments of $1 \mathrm{ml}$ or alfentanil in increments of $200 \mu \mathrm{g}$ according to clinical requirements. If apnoea occurred (defined as no inspiration for at least 30 seconds) respiration was assisted manually. In the halothane group induction was with methohexitone and the patients breathed $66 \%$ nitrous oxide in oxygen. Halothane was given as required during maintenance. Recovery of consciousness was assessed by noting the time taken from discontinuation of nitrous oxide for the patient to respond correctly to "What is your name?" followed by "Show me your left thumb."

\section{STATISTICAL ANALYSIS}

All data are quoted as mean and standard error of the mean (SEM). The $2 \times 2 \chi^{2}$ test was used when comparing sex, ASA grade, and incidence of apnoea. The unpaired Student's $t$ test was used for all other variables. The two indices of recovery showed a skewed distribution, and statistical analysis was therefore performed on logarithmically transformed data.

\section{Results}

Of the 907 patients who entered the study, 643 were included in the analysis; 297 were given alfentanil and 346 halothane. The remaining patients were excluded because either the anaesthetist did not follow the protocol (219 cases) or the record form was incomplete (45).

Full details of the patients, types of surgery, and course of the anaesthetic are available on request to the authors. An extended summary of the data is also available on PRESTEL, page 7104. The treatment groups were com- parable. The mean durations of anaesthesia were 10.92 (SEM 0.45) minutes (alfentanil) and $11.95(0.33)$ minutes (halothane). During maintenance, 211 $(71 \%)$ of the patients given alfentanil required increments of the drug, the first after a mean of 4.88 (SEM 0.21) minutes. Apnoea requiring manually assisted ventilation occurred more frequently in the alfentanil group. At induction 56 patients (19\%) given alfentanil became apnoeic compared with only tive of the patients $(1.4 \%)$ given halothane $(p<0.001)$. Apnoea during maintenance occurred in $34(11.5 \%)$ and one $(0.3 \%)$ of the patients in the two treatment groups, respectively $(p<0.001)$.

The table summarises the recovery data. Recovery was significantly faster in the alfentanil group, the patients responding with their correct name in less than half the time taken by the halothane group $(p<0.001)$. Similar significant differences were seen in the time taken to show the left thumb, recovery times being 4.21 (SEM 0.25$)$ minutes and $9.46(0.29)$ minutes, respectively. Premedication had a pronounced effect on both indices of recovery time, lorazepam delaying recovery more than temazepam.

Mean recovery times (minutes) from discontinuation of nitrous oxide to giving correct name. (SEM in parentheses)

\begin{tabular}{|c|c|c|c|c|}
\hline & \multicolumn{3}{|c|}{ Premedication } & \multirow[b]{2}{*}{ All patients } \\
\hline & Lorazepam & Temazepam & None & \\
\hline Alfentanil & $\begin{array}{c}4.96(0.59) \\
{[n=92]}\end{array}$ & $\begin{array}{l}3.45(0 \cdot 29) \\
{[n=150]}\end{array}$ & $\begin{array}{c}2 \cdot 58(0 \cdot 26) \\
{[n=55]}\end{array}$ & $\begin{array}{c}3 \cdot 75(0 \cdot 24) \\
{[\mathrm{n}=297]}\end{array}$ \\
\hline Halothane & $\begin{array}{c}9.97(0.66) \\
(n=112]\end{array}$ & $\begin{array}{c}8.49(0.39) \\
{[n=166]}\end{array}$ & $\begin{array}{c}6.44(0.41) \\
{[n=68]}\end{array}$ & $\begin{array}{c}8 \cdot 57(0 \cdot 29) \\
{[n=346]}\end{array}$ \\
\hline Alfentanil $v$ halothane & $\mathrm{p}<0.001$ & $\mathrm{p}<0.001$ & $\mathrm{p}<0.001$ & $\mathrm{p}<0.001$ \\
\hline
\end{tabular}

\section{Discussion}

\section{ADVANTAGES OF VIEWDATA}

Viewdata collection and transmission of clinical trial data minimised the problems of handling a large volume of information from 39 geographically well separated centres in Great Britain. Classically a trial monitor would visit each participant several times during the study, a process costly of time, much of it wasted in travel. The opportunity to cut down on these visits was a major reason for using viewdata technology. Costs of travel thus saved must be set against the fee for installing PRESTEL in each centre (£15) and the quarterly PRESTEL bill (including rental: roughly £30). During the study Janssen paid these costs and the telephone connections were routed, with permission, through the hospital switchboard.

A particular cost saving feature of the system allows the use of local, as opposed to trunk, rates for the telephone connection of most PRESTEL terminals to the central computer. Regrettably, the most useful telephone based feature of the system, the electronic "mailbox," entailed trunk calls to London. This telephone message service was shortly to be made available to most subscribers on a local call basis. The electronic mailbox allows each

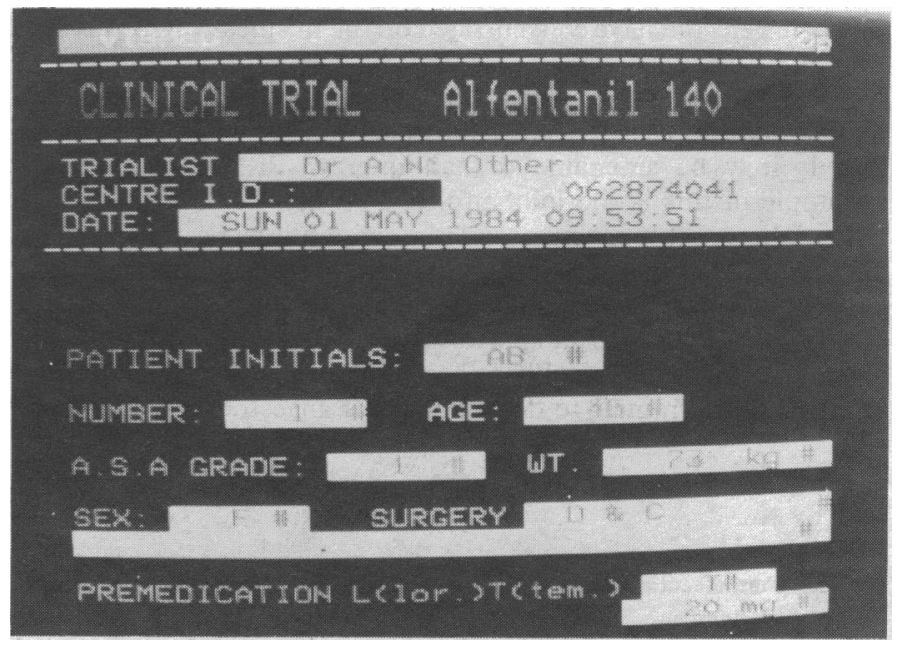

FIG 2-PRESTEL frame showing typical example of a patient record form. 
user to leave messages for any other user at a time that is convenient for the sender. The recipient is alerted to waiting messages whenever he connects his terminal to PRESTEL. During this study extensive use of this facility by the anaesthetists and trial monitor permitted rapid trouble shooting, providing the clinician with convenient, fast responses to his queries and requests.

By providing clear, typewritten record forms to the central coordinator, the viewdata system avoids the problems of ambiguities and misunderstandings which may result from illegible handwriting. This proved of great benefit in checking data and identifying violations of protocol. In addition, the electronic nature of the system provides the twin advantage of speed and confidentiality when compared with the postal system. Rapid data collection gave a useful indicator on a daily basis of the rate of progress for each centre in the study. The daily updating feature of the system might be of major advantage in trials with a sequential analytical design. To maintain confidentiality each participant was made a member of a closed user group, and only these people could summon the trial protocol and record forms. Individual patients were identified by their initials only, together with a trial number (fig 2 ). In addition, only the central coordinator could obtain access to the data once transmitted. Each centre thus proceeded independently without knowing the results obtained by the other trialists.

\section{DISADVANTAGES AND IMPROVEMENTS TO SYSTEM}

Enthusiasm for new technology was a major motivating force for many of the investigators, as well as ourselves, but some were daunted by the apparent complexity of this substitute for pen and paper. Nevertheless, no trialist gave incompatibility with the system as a reason for not cooperating. The small keyboard was the feature that attracted most criticism, requiring considerable time and effort to use. Microcomputers, such as the BBC model B, which are compatible with PRESTEL and have traditional full size keyboards are easier to use but more expensive.

Although the electronic mailbox allows rapid response to specific queries, the system requires further adaptation to check data as they are entered. Facilities are available to connect PRESTEL users to on line computers in order to check entries such as age, weight, or drug dosage against preset limits and to reject values outside the specified range. The clinicians' attention can then be drawn before transmission to obvious errors and items requiring additional explanation, such as violations of protocol. In this study this would have avoided record forms being sent when the patient had received the wrong dose of alfentanil (123 forms) or premedication (63 forms). Many incomplete forms (45 in this study) could also have been brought to the attention of the clinician by allowing access to a subsequent question only after completion of a previous one. As this was a preliminary assessment of the use of viewdata, we did not use this more expensive facility which links the clinician by way of the PRESTEL system to another large mainframe computer. This, however, would also have allowed direct analysis of the cumulated data electronically, without the intermediacy of a trials monitor and the possibility of additional human errors.

A major problem was the separation of the patient in the operating theatre from the terminal connected to the departmental telephone. This necessitated transmitting data separately after recording, increasing the workload of the clinician and introducing the possibility of transcription errors. Geographical separation also prevented the anaesthetist from checking the viewdata system for the next code for random allocation. For most clinical settings the clinician could enter data on to PRESTEL as they were collected, obtain a printout for his own records at the same time, and discover the randomisation code for the next patient.

The system would allow patients to enter and transmit their own data from a computerised questionnaire, but this might be costly of computer and telephone time and would not allow the clinician to check the data before transmission. Although entry of data by patients may be problematical, computers do have a role in taking personal histories and in self assessment. ${ }^{3}$ Carr and coworkers ${ }^{4}$ found good correlation between personal histories from psychiatric patients taken by microcomputer and information recorded by the doctor. In addition, histories elicited by computer disclosed important items previously unknown to the clinician. They also reported successful self rating of depression by computer questionnaire, together with favourable comments from patients, nurses, and medical staff. ${ }^{5}$

The results of this study confirm that the dosage schedule of alfentanil used had the advantage of a more rapid return to consciousness and orientation compared with the more traditional halothane anaesthesia. We would certainly consider the use of viewdata for collecting record forms in future multicentre studies. We recommend including data validation during entry and direct analysis incorporated electronically to avoid transcription errors to use the system to its full advantage. Each department during the trial could use the system to access the full range of PRESTEL services, which include an increasing amount of medical information. On completing the study most of the centres decided to keep the PRESTEL facility for general departmental use. Viewdata equipment is expensive to buy, but as the system becomes more widely available this method of data collection will compete with traditional methods.

We thank Sir Richard Doll and Dr G R Venning for helpful comments, Miss L Leslie for statistical work, and Miss J Alexander for help in preparing the manuscript. We are also grateful to the following anaesthetists who carried out the study and cooperated in establishing the PRESTEL system and making it work: S Allen, K Birkinshaw, D Burt, P Cartwright, N Coote, J Curran, D Desgrand, R Dunnill, H Fischer, A Florence, H Freedman, J Fryer, N Goodman, H Gordon, J Henney, G Hibbert, J Jellicoe, $M$ Johnson, R Johnston, S Jothilingam, B Kay, S Lowe, $\mathrm{K}$ Macleod, J Martin, A MacDonald, A Mellon, C Nixon, U Plantevin, E Proctor, J Sear, G Smith, C Spanswick, J Thorn, S Tinloi, M Watt, G Weetch, E Welchew, J Windsor, C Wright.

\section{References}

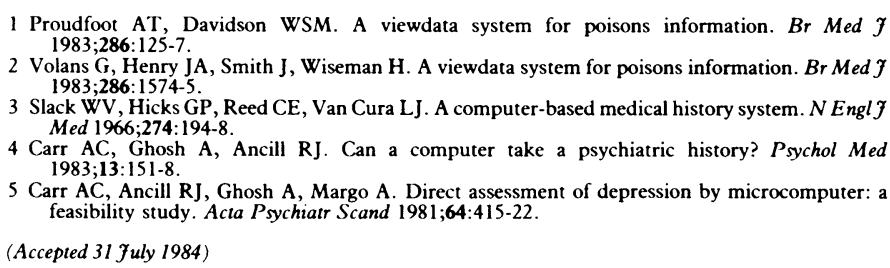

\section{How frequent is postinfluenzal depression?}

Although it has generally been believed that influenza, and other common viral diseases such as infectious mononucleosis and hepatitis, are followed by an increased incidence of depression, hard evidence of the link and its frequency is lacking. ${ }^{1}$ Part of the problem lies in the resemblance between the physical debility and fatigue that may follow some viral and bacterial infections and the more clear cut affective symptoms of mild depression. There is a borderland of mild dysphoria, in which it is hard to make distinctions, particularly in retrospect. A recent study of psychiatric patients found no evidence of increased influenzal antibody titres in depressive illness, ${ }^{2}$ although a follow up of patients with infectious mononucleosis showed an increase in symptoms of mild depression and anxiety. ${ }^{3}$ Nevertheless, clinical experience suggests that after influenza there is an increased incidence of a more severe depression, which contrasts with the milder symptoms that may follow other infections. There may also be a previous or family history of depression, suggesting that the patient is predisposed. Both the clinical picture, which may show pronounced guilt and suicidal risk, and the response to treatment resemble those of other severe endogenous depressions.-E S PAYKEL, professor of psychiatry, London.

' Whitlock FA. Symptomatic affective disorders. A study of depression and mania associated with physical disease and medication. Sydney: Academic Press, 1982 Sinanan K, Hillary I. Post-influenzal depression. Br F Psychiatry 1981 ;138:131-3. Cadie M, Nye FJ, Storey P. Anxiety and depression after infectious mononucleosis
Brf Psychiatry 1976;128:559-61. 